\title{
ON CLEAN LAURENT SERIES RINGS
}

\author{
YIQIANG ZHOU and MICHAŁ ZIEMBOWSKI ${ }^{\bowtie}$
}

(Received 25 January 2013; accepted 14 May 2013; first published online 18 July 2013)

\author{
Communicated by B. Gardner
}

\begin{abstract}
Here we prove that, for a 2-primal ring $R$, the Laurent series ring $R((x))$ is a clean ring if and only if $R$ is a semiregular ring with $J(R)$ nil. This disproves the claim in K. I. Sonin ['Semiprime and semiperfect rings of Laurent series', Math. Notes 60 (1996), 222-226] that the Laurent series ring over a clean ring is again clean. As an application of the result, it is shown that, for a 2-primal ring $R, R((x))$ is semiperfect if and only if $R((x))$ is semiregular if and only if $R$ is semiperfect with $J(R)$ nil.
\end{abstract}

2010 Mathematics subject classification: primary 16S99; secondary 16U99, 16E50, 16L30.

Keywords and phrases: clean ring, Laurent series ring, semiregular ring, semiperfect ring, 2-primal ring, nil Jacobson radical.

\section{Introduction}

Throughout rings are associative with identity. For a ring $R, J(R)$ and $U(R)$ denote the Jacobson radical and the set of units of $R$, respectively. The ring of Laurent series $R((x))$ is defined as a ring that consists of the formal series

$$
\sum_{i=l}^{\infty} a_{i} x^{i}, \quad l \in \mathbb{Z}, a_{i} \in R,
$$

where $x$ is an independent variable, with the ordinary addition and with multiplication given by the formula

$$
\left(\sum_{i=p}^{\infty} a_{i} x^{i}\right)\left(\sum_{j=q}^{\infty} a_{j} x^{j}\right)=\sum_{k=p+q}^{\infty}\left(\sum_{i+j=k} a_{i} b_{j}\right) x^{k} .
$$

The research of the first author was supported by a Discovery Grant from NSERC of Canada, and that of the second author by the Foundation for Polish Science - HOMING PLUS Programme, HOMING PLUS BIS/2011-3/2.

(C) 2013 Australian Mathematical Publishing Association Inc. 1446-7887/2013 \$16.00 
We would like to point out that each element of $R((x))$ can be written as $x^{l} \sum_{i=0}^{\infty} a_{i} x^{i}$ or $x^{l}\left(a_{0}+a_{1} x+\cdots\right)$.

An element of a ring $R$ is called a clean element if it is the sum of an idempotent and a unit, while the ring $R$ is called a clean ring if each of its elements is a clean element (see [4]). A ring $R$ is called 2-primal if the prime radical $N(R)$ is exactly the set of nilpotent elements. Here we prove that, for a 2-primal ring $R, R((x))$ is a clean ring if and only if $R$ is a semiregular ring with $J(R)$ nil. This disproves the claim in [7, Corollary, page 224] that the Laurent series ring over a clean ring is again clean. As an application of the result, it is shown that, for a 2-primal ring $R, R((x))$ is semiperfect if and only if $R((x))$ is semiregular if and only if $R$ is semiperfect with $J(R)$ nil.

\section{The results}

Recall that a reduced ring is a ring with no nonzero idempotents.

Lemma 2.1. Let $R$ be a reduced ring. If $f^{2}=f \in R((x))$, then $f \in R$.

Proof. Suppose that $f=\sum_{i \geq k} a_{i} x^{i}$ is an idempotent of $R((x))$ with $a_{k} \neq 0$. Then

$$
\sum_{i \geq k} a_{i} x^{i}=\left(\sum_{i \geq k} a_{i} x^{i}\right)\left(\sum_{i \geq k} a_{i} x^{i}\right),
$$

which implies that $k=0$ since $R$ is reduced. Suppose that there exists $l>0$ such that $a_{l} \neq 0$. We can assume that $l$ is the smallest with this condition. Thus $a_{i}=0$ for all $0<i<l$, and $f=a_{0}+a_{l} x^{l}+a_{l+1} x^{l+1}+\cdots$. From $f^{2}=f$, it follows that $a_{0}^{2}=a_{0}$ and $a_{l}=a_{0} a_{l}+a_{l} a_{0}$. Multiplying the last equation on the left by $a_{0}$ we have

$$
a_{0} a_{l}+a_{0} a_{l} a_{0}=a_{0} a_{l}
$$

and so $a_{0} a_{l} a_{0}=0$. Since $R$ is reduced, $a_{0} a_{l}=a_{l} a_{0}=0$ and $a_{l}=0$ follows, which is a contradiction. Thus $f=a_{0} \in R$.

Lemma 2.2. Let $R$ be a reduced ring such that $R((x))$ is a clean ring. Then $R$ is (von Neumann) regular.

Proof. Let $a \in R$. Then $-a x^{-1}=e+u$ where $e^{2}=e \in R((x))$ and $u$ is a unit of $R((x))$. By Lemma 2.1, $e \in R$. So $a x^{-1}+e$ is a unit of $R((x))$, and hence $a+e x$ is a unit of $R((x))$. There exists $\sum_{i \geq 0} a_{i} x^{i} \in R((x))$ such that

$$
(a+e x)\left(a_{0}+a_{1} x+\cdots\right)=x^{k}
$$


where $k \geq 0$. Thus, it follows that

$$
\begin{aligned}
a a_{0} & =0 \\
a a_{1}+e a_{0} & =0 \\
a a_{2}+e a_{1} & =0 \\
\vdots & \\
a a_{k-1}+e a_{k-2}= & 0 \\
a a_{k}+e a_{k-1} & =1 .
\end{aligned}
$$

Multiplying the second equation by $a$ from the left yields $a^{2} a_{1}=0$, and this implies $a a_{1}=0$ as $R$ is reduced. A simple induction shows that $a a_{1}=a a_{2}=\cdots=a a_{k-1}=0$. So $a_{k-1} a=0$. Now multiplying the last equation by $a$ from the right gives $a=a a_{k} a$.

Lemma 2.3. Let $a \in R$ and $e^{2}=e \in R$ such that both $e a(1-e)$ and $(1-e)$ ae are contained in $J(R)$. If eae is clean in eRe and $(1-e) a(1-e)$ is clean in $(1-e) R(1-e)$, then a is clean in $R$.

Proof. Write $e a e=u+f$ and $(1-e) a(1-e)=v+h$, where $u \in U(e R e), f^{2}=f \in e R e$, $v \in U((1-e) R(1-e))$ and $g^{2}=g \in(1-e) R(1-e)$. Then $f+g$ is an idempotent of $R$ and $u+v \in U(R)$. As $e a(1-e),(1-e) a e \in J(R),(u+v)+(e a(1-e)+(1-e) a e) \in$ $U(R)$. So, by the Pierce decomposition,

$$
\begin{aligned}
a & =e a e+e a(1-e)+(1-e) a e+(1-e) a(1-e) \\
& =(f+g)+[(u+v)+(e a(1-e)+(1-e) a e)]
\end{aligned}
$$

is a clean element of $R$.

Recall that the prime radical $N(R)$ of a ring $R$ is defined to be the intersection of all the prime ideals in $R$. A 2-primal ring is a ring $R$ such that the prime radical $N(R)$ is exactly the set of nilpotent elements. It is known that a ring $R$ is 2-primal if and only if $R / I$ is a domain for every minimal prime ideal $I$ of $R$. Examples of 2-primal rings include commutative rings and reduced rings.

For a subset $S$ of a ring $R$, let $S((x))$ be the set of Laurent series with coefficients in $S$.

Lemma 2.4. Let $R$ be a 2-primal ring. Then $J(R((x)))=N(R)((x))$.

Proof. If $R$ is 2-primal, then $R / N(R)$ is reduced, and hence $J(R((x)))=N(R)((x))$ by [8, Theorem 2].

Exchange rings were introduced by Warfield [9] via the exchange property of modules. Here we use an equivalent condition of an exchange ring obtained independently by Goodearl-Warfield [1] and Nicholson [4]: a ring $R$ is an exchange ring if and only if for each $a \in R$ there exists $e^{2}=e \in R$ such that $e \in a R$ and $1-e \in$ $(1-a) R$. By [4], every clean ring is an exchange ring, and the converse holds if all idempotents of the ring are central. 
We are now ready to prove the main theorem of the paper. Recall that a ring is said to be strongly regular if it is reduced and regular.

THEOREM 2.5. Let $R$ be a 2-primal ring. The following assertions are equivalent:

(1) $R((x))$ is a clean ring;

(2) $R((x)) / J(R((x)))$ is a clean ring;

(3) $R((x))$ is an exchange ring;

(4) $R((x)) / J(R((x)))$ is an exchange ring;

(5) $R$ is semiregular with $J(R)$ nil;

(6) $R / J(R)$ is strongly regular with $J(R)$ nil.

Proof. (1) $\Rightarrow(2) \Rightarrow(4)$ and (1) $\Rightarrow(3) \Rightarrow(4)$. These are clear.

$(4) \Rightarrow(5)$. By Lemma 2.4, $J(R((x)))=N(R)((x))$. So

$$
(R / N(R))((x)) \cong R((x)) / N(R)((x))=R((x)) / J(R((x)))
$$

is an exchange ring. Since $R$ is 2-primal, $R / N(R)$ is reduced; so all idempotents of $(R / N(R))((x))$ are central by Lemma 2.1. Thus $(R / N(R))((x))$ is a clean ring by $[4$, Proposition 1.8]. Hence $R / N(R)$ is regular by Lemma 2.2. It follows that $J(R) \subseteq N(R)$. So $J(R)=N(R)$, which is nil.

$(5) \Rightarrow(6)$. As $R$ is 2-primal, the assumption implies $J(R)=N(R)$, so $R / J(R)$ is reduced and regular.

$(6) \Rightarrow(1)$. In view of Lemma 2.4, (6) implies $J(R((x)))=J(R)((x))$.

Let $f \in R((x))$, and write $f=x^{-k}\left(a_{0}+a_{1} x+\cdots\right)$, where $k \geq 0$. We show next that $f$ is clean in $R((x))$. As $\bar{R}:=R / J(R)$ is strongly regular, $\bar{a}_{0}=\bar{e}_{0} \bar{u}_{0}$, where $\bar{e}_{0}$ is a central idempotent of $\bar{R}$ and $\bar{u}_{0}$ is a unit of $\bar{R}$. As idempotents of $\bar{R}$ can be lifted to idempotents of $R$, we can assume that $e_{0}^{2}=e_{0}$. Thus, $a_{0}=e_{0} u_{0} e_{0}+j_{0}$, where $e_{0} u_{0} e_{0}$ is a unit of $e_{0} R e_{0}$ and $j_{0} \in J(R)$. Then

$$
e_{0} f e_{0}=x^{-k}\left(\left(e_{0} u_{0} e_{0}+e_{0} j_{0} e_{0}\right)+e_{0} a_{1} e_{0} x+e_{0} a_{2} e_{0} x^{2}+\cdots\right)
$$

is a unit (and hence a clean element) of $\left(e_{0} R e_{0}\right)((x))\left(=e_{0} R((x)) e_{0}\right)$. Since $\bar{e}_{0}$ is central in $\bar{R}, e_{0} f-f e_{0} \in J(R)((x))=J(R((x)))$. So

$$
e_{0} f\left(1-e_{0}\right),\left(1-e_{0}\right) f e_{0} \in J(R((x))) .
$$

By Lemma 2.3, to show that $f$ is clean in $R((x))$ it suffices to show that $\left(1-e_{0}\right) f\left(1-e_{0}\right)$ is clean in $\left(1-e_{0}\right) R\left(1-e_{0}\right)((x))\left(=\left(1-e_{0}\right) R((x))\left(1-e_{0}\right)\right)$. Note that

$$
\begin{aligned}
f_{1} & :=\left(1-e_{0}\right) f\left(1-e_{0}\right) \\
& =x^{-k}\left(\left(1-e_{0}\right) j_{0}\left(1-e_{0}\right)+\left(1-e_{0}\right) a_{1}\left(1-e_{0}\right) x+\cdots\right) .
\end{aligned}
$$

As $R_{1}:=\left(1-e_{0}\right) R\left(1-e_{0}\right)$ is again 2-primal, strongly regular modulo $J\left(R_{1}\right)$ with $J\left(R_{1}\right)$ nil, as argued above we have $\left(1-e_{0}\right) a_{1}\left(1-e_{0}\right)=e_{1} u_{1} e_{1}+j_{1}$, where $e_{1}$ is an 
idempotent of $R_{1}$ which is central modulo $J\left(R_{1}\right), e_{1} u_{1} e_{1}$ is a unit of $e_{1} R_{1} e_{1}$ and $j_{1} \in J\left(R_{1}\right)$. We see that, as $e_{1} j_{0} e_{1} \in J\left(R_{1}\right) \subseteq J\left(R_{1}\right)((x))=J\left(R_{1}((x))\right)$,

$$
e_{1} f_{1} e_{1}=x^{-k}\left(e_{1} j_{0} e_{1}+\left(e_{1} u_{1} e_{1}+e_{1} j_{1} e_{1}\right) x+e_{1} a_{2} e_{1} x^{2}+e_{1} a_{3} e_{1} x^{3}+\cdots\right)
$$

is a unit of $R_{1}((x))$. Since $e_{1} a-a e_{1} \in J\left(R_{1}\right)$ for all $a \in R_{1}$, we have $e_{1} f_{1}\left(1-e_{0}-e_{1}\right)$, $\left(1-e_{0}-e_{1}\right) f_{1} e_{1} \in J\left(R_{1}((x))\right)$. So to show that $f_{1}$ is clean in $R_{1}((x))$, by Lemma 2.3 it suffices to show that $f_{2}:=\left(1-e_{0}-e_{1}\right) f_{1}\left(1-e_{0}-e_{1}\right)$ is clean in $\left(1-e_{0}-e_{1}\right) R(1-$ $\left.e_{0}-e_{1}\right)((x))$.

Let us write $\left(1-e_{0}-\cdots-e_{i-1}\right) a_{i}\left(1-e_{0}-\cdots-e_{i-1}\right)=e_{i} u_{i} e_{i}+j_{i}$, where $e_{i}$ is an idempotent of $R_{i}:=\left(1-e_{0}-\cdots-e_{i-1}\right) R\left(1-e_{0}-\cdots-e_{i-1}\right)$ which is central modulo $J\left(R_{i}\right), e_{i} u_{i} e_{i}$ is a unit of $e_{i} R_{i} e_{i}$ and $j_{i} \in J\left(R_{i}\right)$ for $i=1,2, \ldots, k-1$. Let $f_{i}:=\left(1-e_{0}-\right.$ $\left.e_{1}-\cdots-e_{i-1}\right) f\left(1-e_{0}-e_{1}-\cdots-e_{i-1}\right)$ for $i=1,2, \ldots, k-1$. The argument above shows that

$$
\begin{aligned}
& f \text { is clean in } R((x)) \\
& \Leftrightarrow f_{1} \text { is clean in } R_{1}((x)) \\
& \Leftrightarrow f_{2} \text { is clean in } R_{2}((x)) \\
& \quad \vdots \\
& \Leftrightarrow f_{k} \text { is clean in } R_{k}((x)) .
\end{aligned}
$$

Note that $f_{k}=g+h$, where

$$
\begin{aligned}
& g=x^{-k}\left(1-e_{0}-\cdots-e_{k-1}\right)\left(j_{0}+j_{1} x+\cdots+j_{k-1} x^{k-1}\right)\left(1-e_{0}-\cdots-e_{k-1}\right), \\
& h=\left(1-e_{0}-\cdots-e_{k-1}\right)\left(a_{k}+a_{k+1} x+\cdots\right)\left(1-e_{0}-\cdots-e_{k-1}\right) .
\end{aligned}
$$

As $j_{0}, \ldots, j_{k-1} \in J(R), g \in J\left(R_{k}\right)((x))=J\left(R_{k}((x))\right)$. But $h$ is an element of the power series ring $R_{k}[[x]]$. As $R_{k}[[x]]$ is clean by [2, Proposition 5], $h$ is a clean element of $R_{k}[[x]]$. So $h$ is a clean element of $R_{k}((x))$. Thus $h=w+e$, where $w$ is a unit of $R_{k}((x))$ and $e$ is an idempotent of $R_{k}((x))$. As $g \in J\left(R_{k}((x))\right), g+w$ is a unit of $R_{k}((x))$, so $f_{k}=(g+w)+e$ is a clean element of $R_{k}((x))$. The proof is complete.

COROLlaRY 2.6. Let $R$ be a reduced ring. Then $R((x))$ is clean if and only if $R$ is strongly regular.

Thus, for a domain $R, R((x))$ is clean if and only if $R$ is a division ring. If $R=\mathbb{Z}_{(p)}$ is the localization of $\mathbb{Z}$ at a prime $p$, then $R$ is a local domain that is not a division ring, and hence $R((x))$ is not clean. So this is a counter-example to [7, Corollary, page 224].

A ring $R$ is called strongly $\pi$-regular if for each $a \in R$ there exists $n \geq 1$ such that $a^{n} \in a^{n+1} R$. A commutative ring $R$ is strongly $\pi$-regular if and only if $R / J(R)$ is (strongly) regular with $J(R)$ nil (see [3, Exercise 4.15]).

Corollary 2.7. Let $R$ be a commutative ring. Then $R((x))$ is clean if and only if $R$ is strongly $\pi$-regular. 
The following example shows that in Theorem 2.5 the equivalence ' $(1) \Longleftrightarrow(6)$ ' may not hold if the ring $R$ is not a 2-primal ring.

ExAmple 2.8. Let $S$ be any strongly regular ring and $R=\mathbb{M}_{n}(S)$ with $n \geq 2$. Then $R$ is not strongly regular. But $R((x))$ is clean. In fact, $R((x))=\mathbb{M}_{n}(S)((x)) \cong \mathbb{M}_{n}(S((x)))$ (where $\sum_{l \geq k}\left(s_{i j}^{(l)}\right) x^{l} \mapsto\left(\sum_{l \geq k} s_{i j}^{(l)} x^{l}\right)$ is the required isomorphism). One sees that $S((x))$ is clean by Theorem 2.5 , so $\mathbb{M}_{n}(S((x)))$ is clean by [2].

LEMMA 2.9. If $R=R_{1} \times R_{2}$ is a direct product of rings, then $R((x)) \cong R_{1}((x)) \times R_{2}((x))$.

Proof. The map $\sum_{i \geq k}\left(a_{i}, b_{i}\right) x^{i} \mapsto\left(\sum_{i \geq k} a_{i} x^{i}, \sum_{i \geq k} b_{i} x^{i}\right)$ is the required isomorphism from $R((x))$ onto $R_{1}((x)) \times R_{2}((x))$.

In [10, Theorem], it was proved that $R((x))$ being semilocal implies that $R$ is semiperfect with $J(R)$ nil.

THeOREM 2.10. Let $R$ be a 2-primal ring. The following assertions are equivalent:

(1) $R((x))$ is semiperfect;

(2) $\quad R((x))$ is semilocal;

(3) $R((x))$ is semiregular;

(4) $R((x)) / J(R((x)))$ is regular;

(5) $R$ is semiperfect with $J(R)$ nil;

(6) $R / J(R)$ is a finite direct product of division rings with $J(R)$ nil.

Proof. (1) $\Rightarrow(2) \Rightarrow(4)$ and $(1) \Rightarrow(3) \Rightarrow(4)$. These are clear.

$(4) \Rightarrow(5)$. By Lemma 2.4, $J(R((x)))=N(R)((x))$. So

$$
(R / N(R))((x)) \cong R((x)) / N(R)((x))=R((x)) / J(R((x)))
$$

is regular. By [6], the ring $R / N(R)$ is semisimple Artinian. It follows that $J(R)=N(R)$. So (5) holds.

$(5) \Rightarrow(6)$. As $R$ is 2-primal, $R / N(R)$ is reduced, so $J(R)=N(R)$. Since $R$ is semiperfect, it follows that $R / J(R)$ is a finite direct product of division rings.

$(6) \Rightarrow(1)$. By Theorem 2.5, $R((x))$ is clean. So idempotents of the ring $R((x)) / J(R((x)))$ can be lifted to idempotents of $R((x))$. Since $J(R)$ is nil, $J(R)=N(R)$. Hence $J(R((x)))=J(R)((x))$ by Lemma 2.4. Write $R / J(R)=D_{1} \times \cdots \times D_{k}$, where the $D_{i}$ are division rings. So, in view of Lemma 2.9,

$$
\begin{aligned}
R((x)) / J(R((x))) & =R((x)) / J(R)((x)) \cong(R / J(R))((x)) \\
& \cong D_{1}((x)) \times \cdots \times D_{k}((x)),
\end{aligned}
$$

which is semisimple Artinian. So $R((x))$ is semiperfect.

A ring is called uniquely clean if each of its elements can be uniquely expressed as the sum of an idempotent and a unit. It is known that $R$ is uniquely clean if and only if $R[[x]]$ is uniquely clean (see [5, Corollary 10$]$ ). 
Proposition 2.11. The Laurent series ring $R((x))$ is not uniquely clean for any nontrivial ring $R$.

Proof. If $T:=R((x))$ is uniquely clean, then $T / J(T)$ is boolean by [5, Theorem 20]. So $\overline{1}$ is the only unit of $T / J(T)$. Thus, $\bar{x}=\overline{1}$ in $T / J(T)$, that is, $1-x \in J(T)$. But $1-x$ is clearly a unit of $T$. This is a contradiction.

\section{References}

[1] K. R. Goodearl and R. B. Warfield Jr, 'Algebras over zero-dimensional rings', Math. Ann. 223 (1976), 168-187.

[2] J. Han and W. K. Nicholson, 'Extensions of clean rings', Comm. Algebra 29(6) (2001), 2589-2595.

[3] T. Y. Lam, Exercises in Classical Ring Theory, Problem Books in Mathematics (Springer, Berlin-Heidelberg-New York, 1995).

[4] W. K. Nicholson, 'Lifting idempotents and exchange rings', Trans. Amer. Math. Soc. 229 (1977), 269-278.

[5] W. K. Nicholson and Y. Zhou, 'Rings in which elements are uniquely the sum of an idempotent and a unit', Glasg. Math. J. 46 (2004), 227-236.

[6] K. I. Sonin, 'Regular rings of skew Laurent series', Fundam. Prikl. Mat. 1(2) (1995), 565-568.

[7] K. I. Sonin, 'Semiprime and semiperfect rings of Laurent series', Math. Notes 60 (1996), 222-226.

[8] A. A. Tuganbaev, 'The Jacobson radical of the Laurent series ring', J. Math. Sci. 149(2) (2008), 1182-1186.

[9] R. B. Warfield Jr, 'Exchange rings and decompositions of modules', Math. Ann. 199 (1972), 31-36.

[10] M. Ziembowski, 'Laurent series ring over semiperfect ring can not be semiperfect', Comm. Algebra, to appear, doi:10.1080/00927872.2012.720324.

YIQIANG ZHOU, Department of Mathematics and Statistics, Memorial University of Newfoundland, St.John's, Canada Nfld A1C 5S7

e-mail: zhou@mun.ca

MICHAŁ ZIEMBOWSKI, Faculty of Mathematics and Information Science, Warsaw University of Technology, 00-662 Warsaw, Poland e-mail: m.ziembowski@mini.pw.edu.pl 